\title{
Imaging of Fractal Profiles
}

\author{
Gerardo Di Martino, Member, IEEE, Antonio Iodice, Senior Member, IEEE, \\ Daniele Riccio, Senior Member, IEEE, and Giuseppe Ruello, Member, IEEE
}

\begin{abstract}
In this paper, a model for radar images of fractal (topologically 1-D) profiles is introduced. A twofold approach is followed: on one hand, we analytically solve the problem whenever small-slope profiles are in order; on the other hand, we present a partly analytical and partly numerical setup to cope with the general-slope case. By means of the analytical approach, we evaluate in closed form both the structure function and the power density spectrum of the radar signal. An appropriately smoothed (physical) fractional Brownian model (fBm) process is employed; its introduction is justified by the finite sensor resolution. A fractal scattering model is employed. It is shown that for a fractal profile modeled as an $\mathrm{fBm}$ stochastic process, the backscattered signal turns out to be strictly related to the associated fractional Gaussian noise process if a small-slope regime for the observed profile can be assumed. In the analytical-numerical framework, a profile with prescribed fractal parameters is first synthesized; then, fractal scattering methods (applicable to wider slope regimes with respect to the previous case) are employed to compute the signal backscattered toward the sensor. Finally, the power density spectrum of the acquired radar image is estimated. The obtained spectra are favorably compared with the theoretical results, and a parametric study is performed to assess the overall method behavior.
\end{abstract}

Index Terms-Electromagnetic scattering, fractals, synthetic aperture radar, radar, radar imaging.

\section{INTRODUCTION}

$\mathbf{I}$ $\mathrm{N}$ THE past decades, remote sensing instruments and corresponding data-processing techniques have been remarkably developed: a huge amount of remote sensing data relevant to any part of the globe is now available. The new generation of imaging sensors provides high-resolution data that could lead to extract very valuable information; value-added products from data obtained by remote sensing platforms can be of key relevance for key applications in agriculture [1], [2], rural and urban planning [3], and disaster monitoring and assessment [4], [5]. As a matter of fact, man-made objects can be identified on radar data with spatial resolution of few meters or less [6], [7]; conversely, as far as geophysical applications are concerned, the retrieval from microwave remote sensing data of reliable and meaningful parameters relevant to observed natural surfaces is an open issue of key importance.

In this paper, we model the microwave imaging process in terms of the natural surface parameters (direct problem); the

Manuscript received March 5, 2009; revised July 31, 2009, December 31, 2009, and February 9, 2010. Date of publication April 8, 2010; date of current version July 21, 2010. This work has been partly supported by the Agenzia Spaziale Italiana (ASI) and Carlo Gavazzi Space (CGS) within the MORFEO project, contract no. 2092A/08/15.

The authors are with the Department of Biomedical, Electronic and Telecommunication Engineering, University of Naples "Federico II," 80125 Naples, Italy (e-mail: gerardo.dimartino@unina.it; iodice@unina.it; daniele. riccio@unina.it; ruello@unina.it).

Digital Object Identifier 10.1109/TGRS.2010.2044661 model is mostly developed in analytical form so that it can also be employed to support the corresponding inverse problem, i.e., the automatic extraction of natural parameters from acquired radar data, or, at least, to assess whether an image-processing technique would preserve the information included in the acquired data. Among different remote sensors, we focus on the imaging ones, which generate synoptic views that provide textural information on the sensed area; this is recognized as valuable information being related to morphological and geological features, land use, and social organization of the observed scene.

The models that we introduce in this paper are based on the fractal geometry. Indeed, it was proved that the fractal geometry is the most appropriate mathematical environment to describe the shape of natural scenes; in addition, this fractal description is provided by employing a minimum number of independent parameters [8], [9]. Among the fractal stochastic surface models, the fractional Brownian model (fBm) provides the best choice for the geometrical description of natural surfaces [8]-[11]. However, fBm mathematical surfaces [9] present a major disadvantage: they are strictly not differentiable. This operation plays a fundamental role in almost any scattering evaluation method, being the scattering process related to the surface slope rather than to the surface height. Hence, to effectively define the derivative of this class of surfaces, we need to introduce physical fractals. The description of the fractal models used throughout this paper is provided in Section II. For the sake of simplicity and clarity, two (reasonable) major assumptions are made in this paper, as discussed in the following two paragraphs.

In this paper, we focus our attention on the (topologically) 1-D problem, assuming an $\mathrm{fBm}$ geometrical model for the observed profile: this simplified environment allows us to present our approach and emphasize the obtained results without hampering the mathematical issues. As a matter of fact, the extension to the (topologically) 2-D case is not straightforward: isotropy issues on fractal surfaces should be taken into account, thus making the mathematical framework quite involved; anyway, this extension is the subject of current investigation by the authors of this work.

The speckle effect is an important peculiarity of microwave images and, more generally, of each coherent imaging system; it is originated from the constructive and destructive interference effects between individual plane waves reflected in each resolution cell, and claims for a specific discussion whenever fractal models are in order [12], [13]. However, in this paper, we focus on the ideal (canonical) case of an infinite number of looks so that the speckle effect can be ignored. This canonical situation is quite well approximated, upon request of popular 
applications, by employing modern very high resolution sensors that can provide, through appropriate averages on the single-look products, nice radar images with a very high number of looks. Anyway, we are also working to consider the single look case, which is the nice step to reach a complete modeling of the microwave imaging process relevant to fractal profiles.

In the open literature, the more exhaustive discussion on the imaging of fractal surfaces is provided in [14] and [15], where the imaging of $\mathrm{fBm}$ surfaces is investigated. The approach therein presented is based on a linear approximation of the image intensity as a function of the partial derivatives of the surface: the underlying hypothesis is that the slopes of the observed surface can be assumed to be small. However, in these previous studies, a Lambertian scattering behavior is postulated. But the choice of the electromagnetic scattering model used to compute the field backscattered from a random rough surface must be related to the geometric model used in describing the surface itself and to the wavelength of the incident electromagnetic field. In particular, theoretical and experimental results [9], [16], [17] demonstrated that the use of adequate scattering fractal models strongly improves the accuracy in the evaluation of the signal backscattered from fractal surfaces at microwaves frequencies. Hence, in this work, appropriate fractal electromagnetic models are used [9], and to the best of our knowledge, this is the only work that present a completely fractal framework to study the imaging of fractal objects.

In particular, we here present a twofold approach that provides, in the small-slope regime, an analytical closed-form solution to the imaging problem; conversely, a model-based numerical setup is used to analyze the general-slope case. In Section III, we present the fractal electromagnetic model used in this paper. In Section IV, we make a very short review of what was already presented in the literature on the imaging of fractal profiles and compare it to our approach. Concerning our approach, we present some relevant results regarding the characteristics of the radar image relevant to a fractal (fBm) profile in the small-slope regime, showing how any radar image can be related to a fractional Gaussian noise (fGn) process [18], which is associated with the fBm process used to describe the profile under analysis. Some notes on the extraction of the profile fractal parameters from one radar image are also presented.

Summarizing, the logical sequence dictating the development of the first part of this paper is given as follows.

1) Solve the problem of the nondifferentiability of the fBm.

2) Find the resulting derivative process power density spectrum, and verify if it exhibits any power-law behavior.

3) Discuss via the power density spectrum if the radar image can be considered as a fractal process to be linked with the profile one.

4) Find the relation between the profile and the image power density spectra. This relation can be exploited for retrieving from the radar data the value of the fractal dimension $D$ relevant to the natural profile.

Finally, in Section V, we introduce a numerical setup that is largely based on models and tools that have been recently presented by the same authors [19]; this setup is first used to positively validate the theoretical results we obtained in the small-slope regime. Furthermore, in the general-slope case, the proposed framework is shown to be a very powerful tool to perform some relevant parametric studies on the validity of the proposed approach. Concluding remarks are reported in Section VI.

\section{Fractal Models}

Fractals are widely considered the most appropriate models to describe natural surfaces [8]-[11]. Within this framework, the $\mathrm{fBm}$ stochastic process is the most used surface fractal model [10], [16], [20]; under some circumstances, fBm realizations can be conveniently obtained by employing the Weierstrass-Mandelbrot (WM) function, which is a continuous but nondifferentiable function [9], [21], [22]. The $\mathrm{fBm}$ is defined in terms of the probability density function of its height increments: a stochastic 1-D process $z(x)$ is an $\mathrm{fBm}$ profile if, for every $x$ and $x^{\prime}$ it satisfies the following relation:

$$
\operatorname{Pr}\left\{z(x)-z\left(x^{\prime}\right)<\bar{\zeta}\right\}=\frac{1}{\sqrt{2 \pi} s \tau^{H}} \int_{-\infty}^{\bar{\zeta}} \exp \left(-\frac{\zeta^{2}}{2 s^{2} \tau^{2 H}}\right) d \zeta
$$

where $\tau=\left|x-x^{\prime}\right|$. Equation (2.1) states that the increments of an $\mathrm{fBm}$ exhibit a (stationary) Gaussian distribution with zero mean and $s \tau^{H}$ standard deviation. Then, in the space domain, the two real parameters that control the fBm behavior are given as follows:

1) $H$, the Hurst coefficient $(0<H<1)$, which is related to the fractal dimension $D$ by means of the relation $D=$ $2-H$

2) $s$, the incremental standard deviation, measured in $\left[\mathrm{m}^{(1-H)}\right]$, i.e., the standard deviation of the profile increments at unitary distance.

The $\mathrm{fBm}$ is not stationary, and its autocorrelation is given by [9]

$$
R\left(x, x^{\prime}\right)=\left\langle z(x) z\left(x^{\prime}\right)\right\rangle=\frac{s^{2}}{2}\left(|x|^{2 H}+\left|x^{\prime}\right|^{2 H}-\left|x^{\prime}-x\right|^{2 H}\right)
$$

where $\langle\cdot\rangle$ stands for the statistical mean.

For a stochastic process with stationary increments, the structure function $V(\tau)$ depends only on the lag $\tau$ and is defined as the mean square increments of elevation points placed at distance $\tau$, i.e.,

$$
V(\tau)=\left\langle\left(z(x)-z\left(x^{\prime}\right)\right)^{2}\right\rangle .
$$

The structure function plot is termed the variogram. By employing (2.1), the structure function of an $\mathrm{fBm}$ profile can be expressed in terms of the fractal parameters $H$ and $s$ as follows:

$$
V(\tau)=s^{2} \tau^{2 H}
$$


that in logarithmic form leads to

$$
\log V(\tau)=2 \log s+2 H \log \tau
$$

Thus, in a log-log plane, a linear behavior is obtained for the structure function, with the slope being equal to $2 H$ and the ordinate intercept to $2 \log s$.

Evaluation of the fBm power density spectrum is definitively not straightforward due to the fBm nonstationarity [9]; a convenient way to proceed would preliminarily require using appropriate space-frequency or space-scale analysis techniques [9], [23]. The former approach is based on the use of the Wigner-Ville spectrum, which provides a space-dependent spectrum that is subsequently averaged over a suitable space interval; the latter method is based on a wavelet space-scale analysis, which provides a scale-dependent spectrum that is subsequently averaged over a suitable scale interval. Independently from the employed method, the same result for the $\mathrm{fBm}$ power density spectrum $S(k)$ is obtained, i.e.,

$$
S(k)=S_{o} k^{-\alpha}
$$

where the spectral domain parameters are related to those of the spatial domain by means of the following relationships [9], [24]:

$$
\begin{aligned}
\alpha & =1+2 H=5-2 D \\
S_{0} & =s^{2} \frac{\pi H}{\cos (\pi H)} \frac{1}{\Gamma(1-2 H)}
\end{aligned}
$$

$\Gamma(\cdot)$ being the Gamma function. From $0<H<1$, we get the constraint $1<\alpha<3$, which defines the range of allowed values for the fBm spectral exponent $\alpha$. Note also that the fBm spectral dependence (2.6) provides a linear plot in a $\log (S)-\log (k)$ plane, whose parameters are related to those of the log-log representation introduced for the variogram.

Then, exploiting the linear dependence in the log-log planes of the fBm power density spectrum $S(k)$ and variogram $V(\tau)$, we can, at least in principle, recover $H$ and $s$ by simply performing a linear regression [19].

The fGn is (formally) defined as the derivative process of the fBm: for the particular case of $H=0.5$, we obtain the derivative of the Brownian motion, i.e., a white Gaussian noise. The mathematical fBm is strictly nondifferentiable, implying that the definition of its derivative process claims for special attention. To this end, a simple approach consists of smoothing the fBm by means of an appropriate kernel and of discarding the high-frequency contributions that are responsible for the $\mathrm{fBm}$ nondifferentiability [18]. Hence, starting from the standard $\mathrm{fBm}$ process $z(x)$, we consider the functional $z_{\varphi}(x)$, which is described as follows:

$$
z_{\varphi}(x)=\int_{-\infty}^{\infty} z\left(x^{\prime}\right) \varphi\left(x-x^{\prime}\right) d x^{\prime}
$$

where $\varphi$ is a test function. In particular, by assuming that $\varphi \in$ $C_{0}^{\infty}(\bar{\Omega})$, with $\Omega \subseteq R$, is an open set, the functional defined by
(2.9) can be seen as a distribution, and the $k$ th derivatives of $z_{\varphi}$ can be rigorously computed as follows [25]:

$$
z_{\varphi}^{(k)}(x)=(-1)^{k} \int_{-\infty}^{\infty} z\left(x^{\prime}\right) \varphi^{(k)}\left(x-x^{\prime}\right) d x^{\prime}
$$

However, an infinitely differentiable kernel is not necessary, for our purposes; hence, for the sake of simplicity, we select $\varepsilon>0$ and pick the following test function:

$$
\varphi(x)= \begin{cases}\frac{1}{\varepsilon}, & \text { if } x \in[0, \varepsilon] \\ 0, & \text { otherwise. }\end{cases}
$$

We incidentally note that at this stage of our presentation, the parameter $\varepsilon$ is measured in meters, it can be arbitrarily set, and it has a clear mathematical meaning; the choice of its value becomes clear whenever its physical meaning is discussed in the following sections.

By substituting (2.11) into (2.10) and computing the first derivative of the process, we can write the derivative of the smoothed process as

$$
z^{\prime}(x ; \varepsilon)=\varepsilon^{-1}[z(x+\varepsilon)-z(x)] .
$$

Hence, the derivative of the smoothed process can be recognized as a finite difference over the original fBm process. This result should not be regarded as the usual finite difference approximation of the derivative, largely employed in engineering applications: in fact, in the majority of cases, its use is justified by the fact that the considered function is regular so that the value of the finite difference is a good approximation of the exact value of the function derivative; conversely, in the case of interest, no exact value of the function derivative exists, with the function being nondifferentiable. Accordingly, (2.12) is not an obvious result and exhibits some useful properties. First, the $z^{\prime}(x ; \varepsilon)$ process turns out to be a stationary Gaussian process, with $\mu=0$ and $\sigma=s \varepsilon^{H-1}$, as can be easily argued from the pdf in (2.1). Moreover, we can evaluate the autocorrelation function as the correlation between two increments of the original $\mathrm{fBm}$ process, i.e.,

$$
\begin{aligned}
R_{z^{\prime}}(\tau ; \varepsilon)= & \left\langle z^{\prime}(x ; \varepsilon) z^{\prime}(x+\tau ; \varepsilon)\right\rangle \\
= & \varepsilon^{-2}\langle z(x+\varepsilon) z(x+\tau+\varepsilon)-z(x+\varepsilon) z(x+\tau) \\
& \quad-z(x) z(x+\tau+\varepsilon)+z(x) z(x+\tau)\rangle .
\end{aligned}
$$

The four terms in the brackets can be evaluated by using the expression of the original profile autocorrelation [see (2.2)]; simple algebra shows that in this case, the terms in (2.2), depending on the position cancel out, and the autocorrelation of the process derivative turns out to be

$$
\begin{aligned}
R_{z^{\prime}}(\tau ; \varepsilon)=\frac{1}{2} s^{2} \varepsilon^{2 H-2} & {\left[\left(\frac{|\tau|}{\varepsilon}+1\right)^{2 H}\right.} \\
& \left.-2\left|\frac{\tau}{\varepsilon}\right|^{2 H}+\left|\frac{|\tau|}{\varepsilon}-1\right|^{2 H}\right] .
\end{aligned}
$$


It is interesting to verify that this function of the space lag asymptotically exhibits an appropriate power-law behavior whenever lags much greater than $\varepsilon$ are taken into account. In fact, for $(\tau / \varepsilon) \gg 1$, by expanding, up to the second order, the first and the last term in the square brackets of (2.14), we obtain

$$
R_{z^{\prime}}(\tau) \cong s^{2} H(2 H-1)|\tau|^{2 H-2} .
$$

For a stationary isotropic stochastic process, the structure function can be derived from the autocorrelation function [9]; in our case, we get

$$
\begin{aligned}
V_{z^{\prime}}(\tau ; \varepsilon)=s^{2} \varepsilon^{2 H-2}[2 & -\left(\frac{|\tau|}{\varepsilon}+1\right)^{2 H} \\
& \left.+2\left|\frac{\tau}{\varepsilon}\right|^{2 H}-\left|\frac{|\tau|}{\varepsilon}-1\right|^{2 H}\right]
\end{aligned}
$$

whose asymptotic behavior for large lags can, again, be emphasized by considering $(\tau / \varepsilon) \gg 1$, i.e.,

$$
V_{z^{\prime}}(\tau ; \varepsilon) \cong 2 s^{2}\left[\varepsilon^{2 H-2}-H(2 H-1)|\tau|^{2 H-2}\right] .
$$

With $z^{\prime}(x ; \varepsilon)$ being a stationary process, we can evaluate its spectrum, by employing the Wiener-Kintchine theorem, as the Fourier transform (FT) of the following autocorrelation function:

$$
\begin{aligned}
S_{z^{\prime}}(k ; \varepsilon)=\frac{1}{2} s^{2} \varepsilon^{2 H-2} & \int_{-\infty}^{\infty}\left[\left(\frac{|\tau|}{\varepsilon}+1\right)^{2 H}\right. \\
& \left.-2\left|\frac{\tau}{\varepsilon}\right|^{2 H}+\left|\frac{|\tau|}{\varepsilon}-1\right|^{2 H}\right] e^{-i k \tau} d \tau .
\end{aligned}
$$

It is very informative (and useful) to evaluate this spectrum in closed form. However, this spectrum does not fall within the class of functions whose FT is known in the Hilbert space of square-summable functions $L^{2}(\Omega)$, where $\Omega \subseteq R$ is an open set. However, to compute the integrals in (2.18), it is possible to use the generalized FT; in particular, the generalized FT of the second term in (2.18) is given by [9], [26]

$$
\int_{-\infty}^{\infty}\left|\frac{\tau}{\varepsilon}\right|^{2 H} e^{-i k \tau} d \tau=-\varepsilon^{-2 H} 2 \Gamma(1+2 H) \sin (\pi H) \frac{1}{|k|^{1+2 H}} .
$$

Using basic FT properties (which are also valid for the generalized FTs), we can easily evaluate the first and third terms in (2.18). Substituting these results into (2.18) and using the Euler formula, we finally get the power density spectrum of the derivative process of the smoothed surface profile, i.e.,

$$
S_{z^{\prime}}(k ; \varepsilon)=2 s^{2} \varepsilon^{-2} \Gamma(1+2 H) \sin (\pi H)(1-\cos (k \varepsilon)) \frac{1}{|k|^{1+2 H}} .
$$

It is interesting to consider how this function asymptotically behaves whenever spatial lags much greater than $\varepsilon$ (i.e., $k \varepsilon \ll$
$2 \pi$ ) are taken into account. In this asymptotic case, (2.20) takes the following relevant form:

$$
S_{z^{\prime}}(k) \cong s^{2} \Gamma(1+2 H) \sin (\pi H) \frac{1}{|k|^{2 H-1}} .
$$

Analysis of (2.20) and (2.21) leads to meaningful results. First, by comparing the spectral exponent in (2.21) to that in (2.6), it turns out that the fGn can be formally assumed to be, asymptotically, a fractal with $H \in[-1,0]$, thus confirming the intuitive remarks obtained in [27]; this formal assumption, although tempting, is not mathematically acceptable because if $H \notin[0,1]$, an associated stochastic process whose fractal dimension is $D=2-H$ cannot be straightforwardly defined [10], [18]. Accordingly, we only conclude that the proposed fGn is a stationary Gaussian process and that it is self-affine of parameter $H$ [10]; due to the obtained $H$ value range, a Hausdorff-Besicovitch fractional dimension for this class of processes cannot simply be defined. Furthermore, the process whose spectrum has been evaluated in (2.20) only asymptotically (i.e., for sufficiently low spatial frequencies) exhibits a spectral power-law behavior. As a matter of fact, as soon as the spatial frequency is of the order or greater than $2 \pi / \varepsilon$, the power density spectrum oscillating behavior begins to dominate.

However, as we have quantitatively shown in this section, the fGn process (which in the following is related to the radar data) somehow inherits the fractal parameters of the original $\mathrm{fBm}$ employed to describe the profile. In Section V, starting from the above presented results, we discuss an imaging model that makes use of the fGn process, and we provide some hints on the retrieving of the $\mathrm{fBm}$ parameters from the corresponding radar amplitude data.

\section{SCATTERING MODELS}

We note that the Lambertian scattering model is not always adequate to describe the electromagnetic scattering from natural surfaces at microwaves: this statement was also proven by theoretical and experimental studies [9], [17]. More specifically, in most of the works available in the literature and dealing with this subject, a heuristic function is used to describe the scattering from an $\mathrm{fBm}$ surface [28], [29]. We believe that each surface geometrical model calls for an appropriate scattering method; hence, if an $\mathrm{fBm}$ model is assumed for the observed surface, then we have to use appropriate fractal-based scattering methods for the evaluation of the scattering, e.g., the small perturbation method (SPM) approximation, the Kirchhoff approach, or the extended boundary condition method [9], [16]; in any case, these fractal scattering methods exhibit a better accuracy, with respect to classical methods, in the evaluation of the field scattered by natural surfaces [9], [17].

Among the fractal-based scattering models available in the literature, we select the SPM because it provides a very simple relation between the physical parameters ( $H$ and $s$ ) of the observed surface and the field backscattered from it. The SPM method is based on the Rayleigh hypothesis and on a surface field series expansion; it can be used for surface height variations that are small compared to the electromagnetic wavelength [30]. A detailed discussion on the validity limits of 
this scattering model is beyond the scope of this work, and it can be found in [9]: we are here mainly interested in using the SPM model.

A monostatic radar is considered, thus, the normalized radar cross section (NRCS) is evaluated. Using the SPM in conjunction with the fBm power density spectrum in (2.6), we obtain the following expression for the NRCS of the profile [24]:

$$
\sigma_{m n}^{0}=4 k^{3} \cos ^{4} \vartheta\left|\beta_{m n}\right|^{2} \frac{S_{0}}{(2 k \sin \vartheta)^{1+2 H}}
$$

where $\vartheta$ is the local incidence angle, $k$ is the electromagnetic wavenumber, and $\beta_{m n}$ are functions of the surface electromagnetic properties and account for polarization issues with respect to the transmitting and the receiving antennas.

Note that the fractal parameters $S_{0}$ and $H$ used in (3.1) rigorously refer to the microscopic description of the profile, i.e., they are those relevant to spatial scales smaller than the radar resolution. As a matter of fact, some natural surfaces may present different fractal parameters at different observation scales (multifractals); this is physically recognized as an effect, at different scales, of different formation mechanisms on the Earth's roughness [8] (wind or sea erosion, tectonic movements, and so on). For the sake of simplicity, in this paper, we focus on fractal profiles presenting the same fractal parameters at every scale. However, some more considerations on this issue will be provided in the following section.

\section{Fractal IMAging Model}

In this section, we present a model for the imaging of fractal profiles whose geometric description is detailed in Section II. The expression for the image intensity can be approximated as done in [15], i.e.,

$$
i(x, y) \cong a_{0}+a_{1} p(x, y)+a_{2} q(x, y)
$$

where $p(x, y)$ and $q(x, y)$ are the partial derivatives of the surface with respect to the range and azimuth directions, respectively, and $a_{0,1,2}$ are the coefficients of the Mac-Laurin series expansion of $i(x, y)$ in terms of $p(x, y)$ and $q(x, y)$. However, $p(x, y)$ and $q(x, y)$ nowhere exist for fractal surfaces, and only a formal use of the partial derivatives of the surface can be proposed [15].

Alternatively, a sufficiently smoothed approximation of the sensed fBm surface, allowing the existence of the partial derivatives, is called for. In remote sensing applications, but more generally, in all engineering matters, the use of the smoothed process is not only desirable, but also necessary. Two different parameters establish the scales of interest for the definition of the physical fractal, i.e., of the considered smoothed process. The first parameter is the wavelength $\lambda$ of the electromagnetic field used to sense the scene: as a matter of fact, objects with size lower than a fraction of this wavelength do not contribute significantly to the generation of the backscattered signal [9], [24]. Accordingly, we can introduce a first smoothing on the surface, where the value of $\varepsilon$ to be included in (2.11) is set equal to a fraction of $\lambda$. However, to set the small scale limits as well, the sensor resolution $\Delta x$ must be taken into account: as a matter of fact, the observed scene is filtered according to the sensor impulse response, and scales smaller than that of the resolution do not significantly contribute to the formation of the final mean intensity image, with their overall effect being apparent only via the speckle effect. This allows us to conveniently employ the fBm smoothed process presented in the Section II: the smoothing process finds here a physical counterpart in the band-limiting operation introduced by the role of both the signal wavelength and the radar resolution. In particular, if $\Delta x \gg \lambda$ as it is the case for radar remote sensing, we can directly work on the following process:

$$
z(x ; \Delta x)=\int_{-\infty}^{\infty} z\left(x^{\prime}\right) \varphi\left(x-x^{\prime}\right) d x^{\prime}
$$

where $\varepsilon$ in (2.11) has been set equal to the sensor resolution cell size $\Delta x$.

If we assume that the slopes of the observed profile are adequately small, we can expand the SPM expression of the backscattering coefficient (3.1) into a Mac-Laurin series. Then, taking into account the above reported considerations on the sensor resolution and the fact that we are considering a (topologically) 1-D profile, we get

$$
i(x ; \Delta x) \cong a_{0}+a_{1} p(x ; \Delta x)
$$

wherein, thanks to the results presented in the previous sections, we can state that $p(x ; \Delta x)$ is the process $z^{\prime}(x ; \Delta x)$ defined in (2.12). Equation (4.3) states that the image inherits the stochastic behavior of this process, i.e., it is Gaussian distributed, with $\mu=a_{0}$ and $\sigma=a_{1} s \Delta x^{H-1}$, as can easily be argued by combining the results obtained in Section II with (4.3). It is interesting to note that the expression in (4.3) allows us also (if it is the case) to introduce a two-scale fractal model, based on two different sets of fractal parameters, at the microscopic and macroscopic scales, respectively. As a matter of fact, derivation of (4.3) shows that while $p(x ; \Delta x)$ is dependent on the fractal parameters at radar resolution scale, the constants $a_{0}$ and $a_{1}$ depend on the fractal parameters of the surface at scales lower than the resolution one, as anticipated in Section III. Note that the presented model, if required, can be used in combination with any scattering diagram: however, only in the fractal case will the coefficients $a_{0,1}$ directly depend on the fractal parameters of the observed profile.

In addition, a combined use of the expression in (4.3) with the results presented in Section II provides the following expressions for the structure function $V_{I}$ and the power density spectrum $S_{I}$ of the image intensity:

$$
\begin{aligned}
& V_{I}(\tau ; \Delta x)=a_{1}^{2} V_{z^{\prime}}(\tau ; \Delta x) \\
& S_{I}(k ; \Delta x)=a_{1}^{2} S_{z^{\prime}}(k ; \Delta x) .
\end{aligned}
$$

These expressions can be rendered in closed form (both in the spatial and the spectral domains) by employing results that we obtained in the previous sections for the structure function and power density spectrum of the derivative of the smoothed profile. In particular, (2.16) and (2.20) must be substituted into (4.4) and (4.5) to get the radar image structure function 


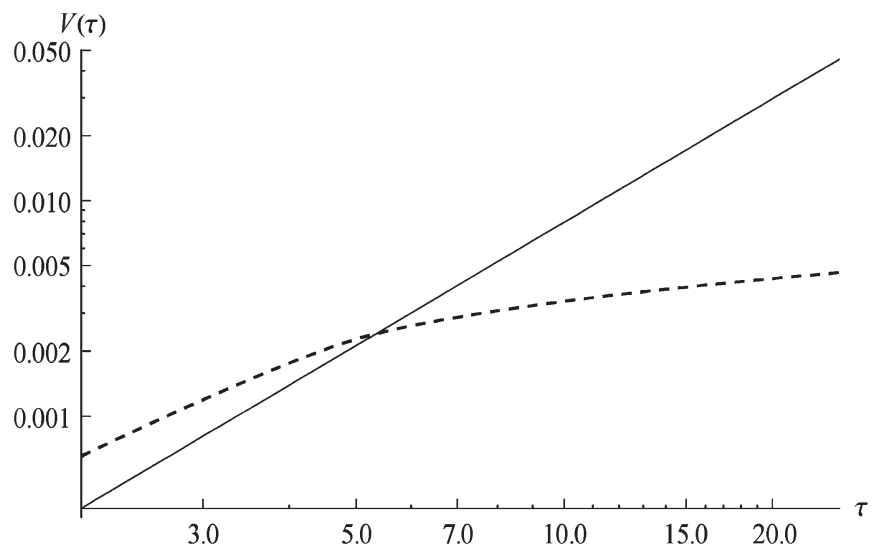

Fig. 1. Log-log variogram of the mathematical fractal profile (full line) and of the derivative of its physical counterpart (dashed line). $H=0.95 ; s=$ $0.01 \mathrm{~m}^{(1-H)} ; \Delta x=5 \mathrm{~m}$; and $a_{1}=10$.

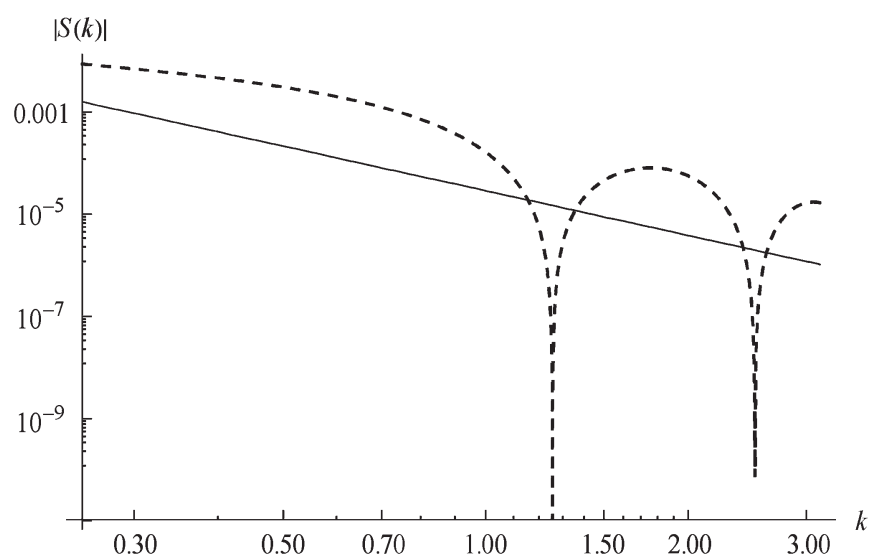

Fig. 2. Log-log plot of the power density spectrum of the mathematical fractal profile (full line) and of the derivative of its physical counterpart (dashed line). $H=0.95 ; s=0.01 \mathrm{~m}^{(1-H)} ; \Delta x=5 \mathrm{~m} ;$ and $a_{1}=10$.

and spectrum, whereas (2.17) and (2.21) must be substituted to get their asymptotic (for large lags or small wavenumbers) counterparts.

Log-log plots of the structure function and power density spectrum of the image are shown in Figs. 1 and 2, respectively; for reference purposes, in each figure, the plots of the corresponding function relevant to the profile are also reported.

A comparison with already existing results is in order. As far as the parameter regulating the power density power-law decay of the image is concerned, we observe that the result shown in (4.5) is analogous to the one obtained in [15] only under the asymptotic limit (small spatial frequencies, i.e., large scales), where $S_{z^{\prime}}$ is provided by (2.21). As a matter of fact, the formal use of the surface derivatives in [15] allows working by rigorously setting $\varepsilon=0$ : the discussion we introduced leads to easily recognize that this setting implies to be always under the regime of validity of the aforementioned asymptotic expressions. In any case, also in the asymptotic limit, we here get a different value, with respect to [15], for the other parameter (the multiplicative constant) characterizing the power density.

More precisely, by looking at (4.5) and (2.20), we can affirm that the spectral behavior of a radar image is, in general, no longer a power-law one and only the case of spatial frequencies for which it is possible to consider (2.21) would suggest a Hurst coefficient of the image $H_{I}$ equal to $H_{T}-1$, with $H_{T}$ being the Hurst parameter relevant to the observed scene. As already mentioned in Section II, this asymptotic result is also in agreement with the observation made in [27], suggesting that the Hurst parameter, upon differentiation, is decreased by one. Nevertheless, as we already argued above, the possibility of defining a fractal dimension for such a process is questionable. Moreover, evaluating the image structure function and power density spectrum allows defining what can be obtained by applying to the radar image any algorithm designed to estimate the fractal parameters of a fractal profile.

Some considerations on the retrieval of the fractal parameters of the observed profile from the image are now in order. As a matter of fact, we can conveniently make use of the relations (4.4), (4.5), (2.16), (2.17), (2.20), and (2.21). As shown in Section II, the fractal parameters estimation techniques for an $\mathrm{fBm}$ are based on one linear regression on $\log -\log$ plots of the variogram or of the power density spectrum. This simple technique cannot be directly used on the image. In particular, it is evident that if we use the general expressions obtained from (2.16) and (2.20), a linear regression in the log-log plane is no longer possible. Anyway, very often for popular radar data, the asymptotic expressions obtained from (2.17) and (2.21) can be used: in this case, the structure function does not exhibit a power-law behavior, while the power density spectrum exhibits this type of behavior for low wavenumbers, which turns out to be the band of interest to recover the fractal parameters via a standard, and simple, linear regression procedure applied in the log-log plane.

\section{NUMERiCAL SETUP}

In this section, a numerical framework based on reliable direct geometric and electromagnetic models is used to analyze the imaging process of a generic 1-D fractal profile without the need of assuming any particular hypothesis on its slope. In this elaboration chain, some models and tools recently presented by the authors are also used [19].

The first step is the generation of the fractal fBm profile. This is achieved by using the WM function as detailed in [9], [21], and [22]. The WM function can be seen as a superposition of an infinite number of sinusoidal tones, i.e.,

$$
z(x)=B \sum_{p=-\infty}^{\infty} C_{p} \nu^{-H p} \sin \left(k_{0} \nu^{p} x+\varphi_{p}\right)
$$

where $B$ (in meters) is an overall amplitude scaling factor, $k_{0}$ (in units per meter) is the wavenumber of the fundamental component, $\nu$ is the seed of the geometrical progression accounting for spectral separation of consecutive components of the surface, $C_{p}$ and $\varphi_{p}$ regulate the amplitude and phase behavior, respectively, of the $p$ th tone, and $H$ is the Hurst coefficient. In order to obtain a random WM function, the coefficients $C_{p}$ and $\varphi_{p}$ have to be random, and the usual choice is a normal distribution for $C_{p}$ and a uniform distribution in $[-\pi, \pi)$ for $\varphi_{p}$.

The WM random function is a predictable random function, and this greatly simplifies the profile synthesis that can be 
TABLE I

List of THE PARAMETERS USED IN THE Simulations AND Summary OF RESUlts

\begin{tabular}{|c|c|c|c|c|c|c|}
\hline & Case 1 & Case 2 & Case 3 & Case 4 & Case 5 & Case 6 \\
\hline Frequency $[\mathrm{GHz}]$ & 1 & 1 & 1 & 1 & 1 & 1 \\
\hline Resolution $[\mathrm{m}]$ & 5 & 5 & 5 & 5 & 20 & 1 \\
\hline Sensor height $[\mathrm{km}]$ & 10 & 10 & 10 & 10 & 40 & 2 \\
\hline Semi-aperture angle $\left[^{\circ}\right]$ & 10 & 10 & 10 & 10 & 10 & 10 \\
\hline Look angle $\left[{ }^{\circ}\right]$ & 10 & 10 & 10 & 10 & 10 & 10 \\
\hline$H$ & 0.95 & 0.55 & 0.95 & 0.55 & 0.55 & 0.55 \\
\hline$s\left[\mathrm{~m}^{1-H}\right]$ & 0.01 & 0.01 & 1 & 1 & 0.01 & 0.01 \\
\hline Expected spectral slope & -0.90 & -0.10 & -0.90 & -0.10 & -0.10 & -0.10 \\
\hline Retrieved spectral slope & -0.92 & -0.09 & -0.69 & -0.07 & -0.09 & -0.08 \\
\hline Squared distance & 0.34 & 0.46 & 0.55 & 0.50 & 0.38 & 0.49 \\
\hline
\end{tabular}

performed by acting on the WM random parameters. The connection between WM functions and $\mathrm{fBm}$ processes can be established by equating the spectral power of the WM and of the $\mathrm{fBm}$ in appropriate spectral intervals [9]. Furthermore, to obtain a physical WM, it is mandatory to limit the summation extent in (5.1) to a finite number of tones, dictated by the minimum and maximum scales of interest, as discussed in Section IV (see also [9]).

Once a realization of the profile has been synthesized, we evaluate its reflectivity. We approximate the profile through facets whose projection on the ground is equal to the sensor resolution $\Delta x$, and for each facet, we evaluate the backscattered signal by using (3.1). As previously mentioned, the geometrical model used in this section is based on the assumption that the observed profile shows the same fractal parameters at all the scales of interest and, in particular, at scales greater or lower than the radar resolution. The inputs for the profile reflectivity evaluation are given in terms of sensor resolution, sensor height, antenna beamwidth, and radar look angle: through these parameters, the simulation software is able to evaluate the extension of the scene and the number of facets.

The final step is the estimation of the power density spectra of the original profile and of the computed backscattered signal; these spectra must be compared with the analytical results presented in closed form in the previous sections. Note that the evaluation of these power-law spectra is not a trivial issue because they are subject to extreme leakage and high variance problems [31]. Among the techniques used to effectively retrieve this type of spectra, we chose the Capon filtering [32]. Nevertheless, the very high and low frequency regions of the estimated spectra cannot be reliably evaluated [32]. The main results of this analysis are presented in the following.

In Table I, we report the parameters used in all the simulations presented hereafter. In all these simulations, the VV polarization case has been assumed: for the employed values of the fractal parameters, any change of the polarization does not significantly affect the obtained results. In all the subsequent figures presenting the simulation results, the plots are appropriately normalized to simplify the comparison of the spectral slopes.

The first elaboration is relevant to a case in which we can safely adopt the small-slope hypothesis [9], [16]. Fig. 3 shows that the profile simulation technique that we employed is a reliable one and that the fit between the estimated and the theoretical spectrum of the image is very good. Similar good

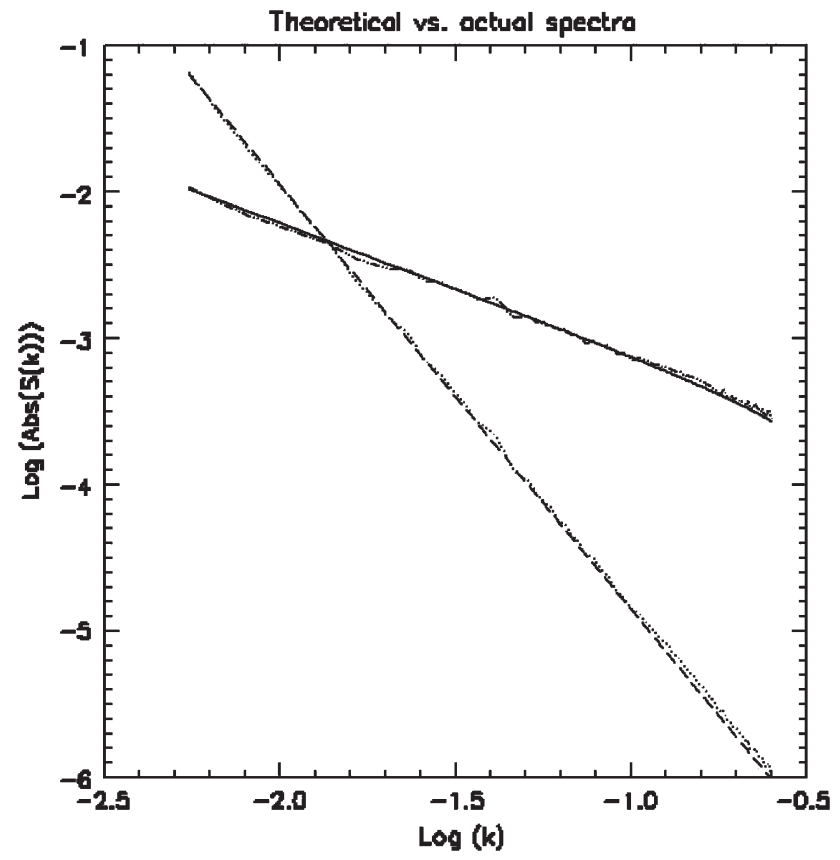

Fig. 3. Theoretical spectra of the surface (long dashed) and of the image (full line) versus the estimated ones (dotted and dash dot, respectively). See case 1 of Table I for the employed parameters.

results hold for different profiles whose corresponding plots are not reported here.

In Figs. 4-6, some relevant simulations for different values of the fractal parameters are reported: in these simulations, the small-slope hypothesis is no longer strictly satisfied [9], [16]. Accordingly, a mismatch between the experimental and theoretical results now appears.

A major result of our paper is to provide the theory that allows retrieving the fractal profile spectral slope by directly working on its microwave image. Within this framework, results in Table I are able to provide quantitative indicators of the agreement between theoretical and experimental results; note that the slope of the obtained spectra is here estimated via a simple linear regression algorithm. Furthermore, in Table I, the goodness of fit between the experimental image power density spectrum and its linear fitting is provided: this is defined as the square distance between the experimental and the fitted curve and can then be interpreted as a quantitative measure of the degree of linearity of the retrieved spectra.

In Figs. 7 and 8, we investigate the role of radar resolution in the imaging process. The lower $\Delta x$ is, the higher the standard 


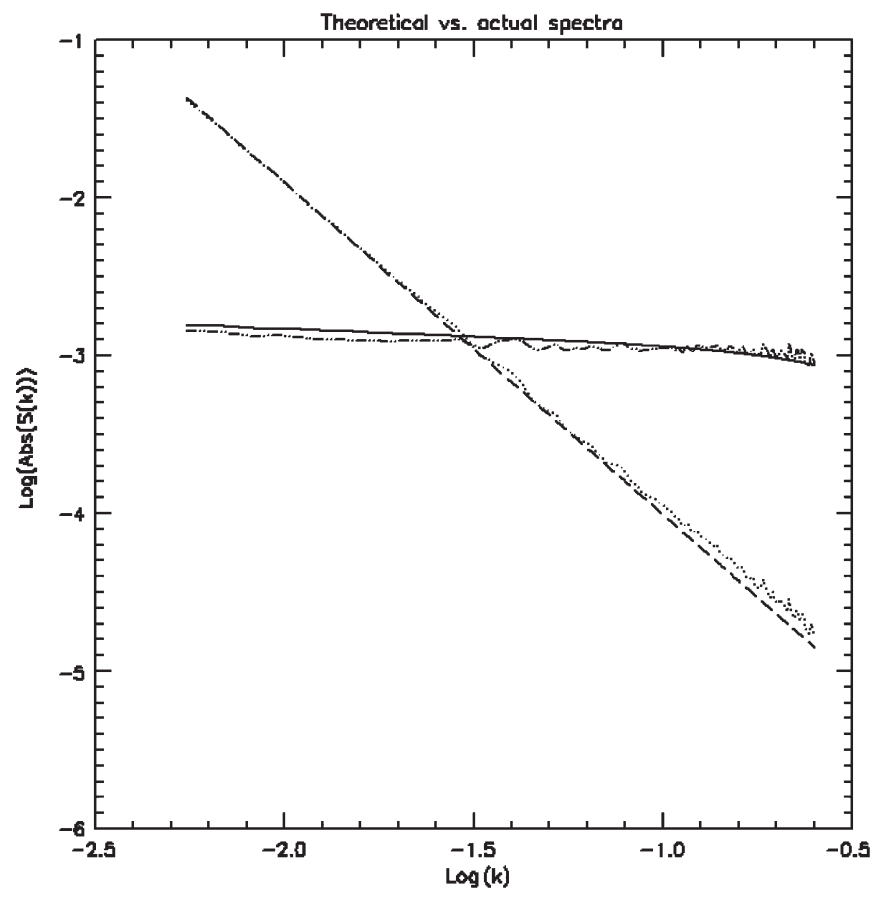

Fig. 4. Theoretical spectra of the surface (long dashed) and of the image (full line) versus estimated ones (dotted and dash dot, respectively). See case 2 of Table I for the employed parameters.

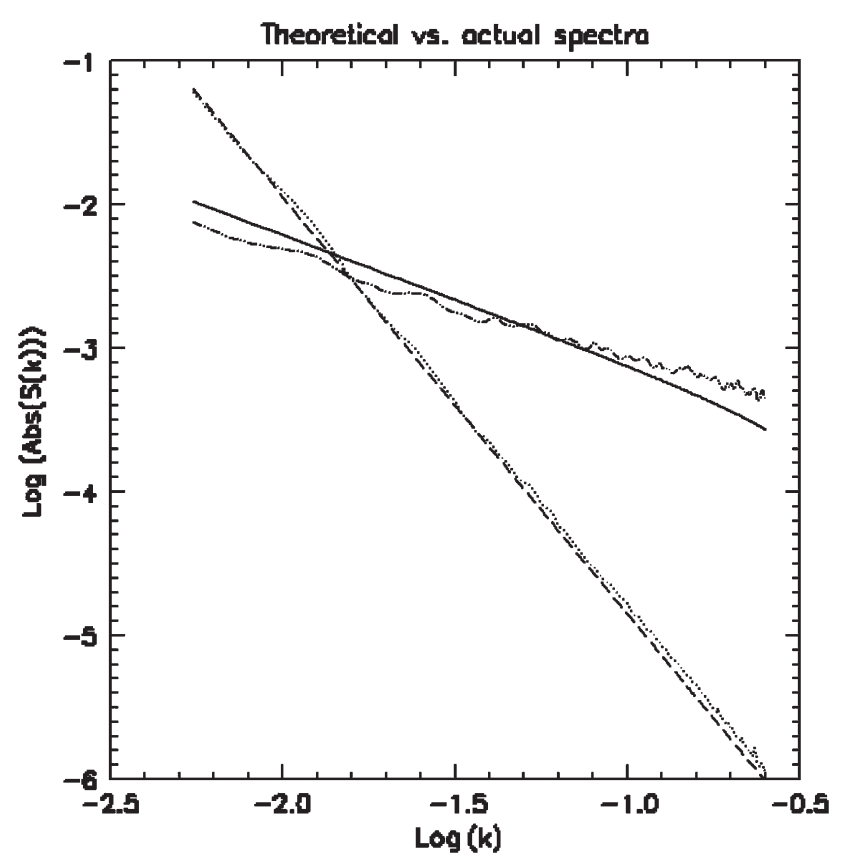

Fig. 5. Theoretical spectra of the surface (long dashed) and of the image (full line) versus estimated ones (dotted and dash dot, respectively). See case 3 of Table I for the employed parameters.

deviation of the slope process will be (i.e., $\sigma=a_{1} s \Delta x^{H-1}$, where the exponent of $\Delta x$ is always negative), where this behavior is more evident for lower values of $H$. Hence, in the two last columns of Table I, we report results obtained for $H=0.55$. Comparison of the goodness of fit values relevant to cases 4,5 , and 6 confirms that the higher the radar resolution is, the more evident the nonlinear behavior exhibited by the image power density spectra will be.

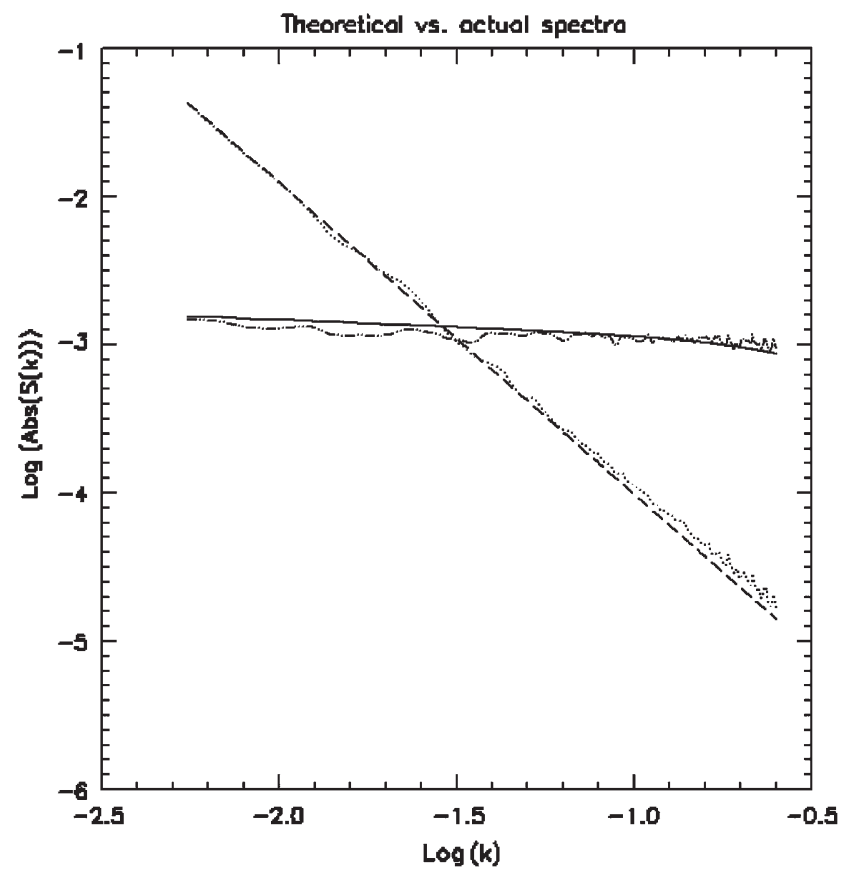

Fig. 6. Theoretical spectra of the surface (long dashed) and of the image (full line) versus estimated ones (dotted and dash dot, respectively). See case 4 of Table I for the employed parameters.

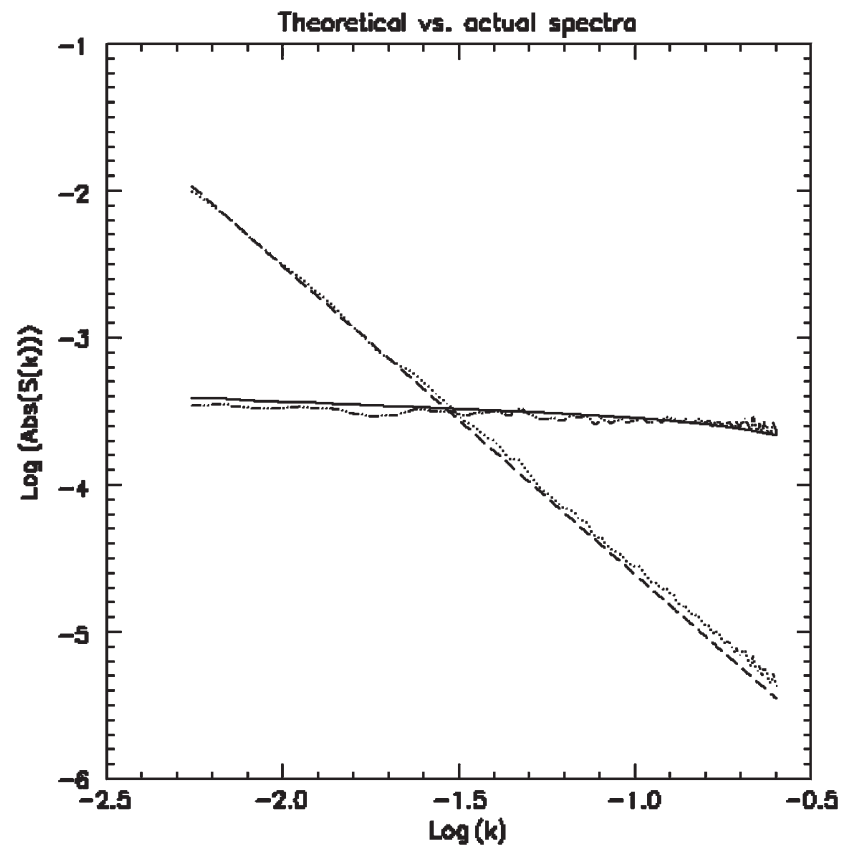

Fig. 7. Theoretical spectra of the surface (long dashed) and of the image (full line) versus estimated ones (dotted and dash dot, respectively). See case 5 of Table I for the employed parameters.

\section{CONCLUSION}

Fractal geometry and scattering models were widely proved to be powerful instruments to represent natural surfaces and their scattering behavior, and their use in all the related applications is quickly increasing.

In this paper, we have presented a mathematical model for microwave imaging of fractal profiles. The model has been obtained in closed form for small-slope profiles, whereas a 


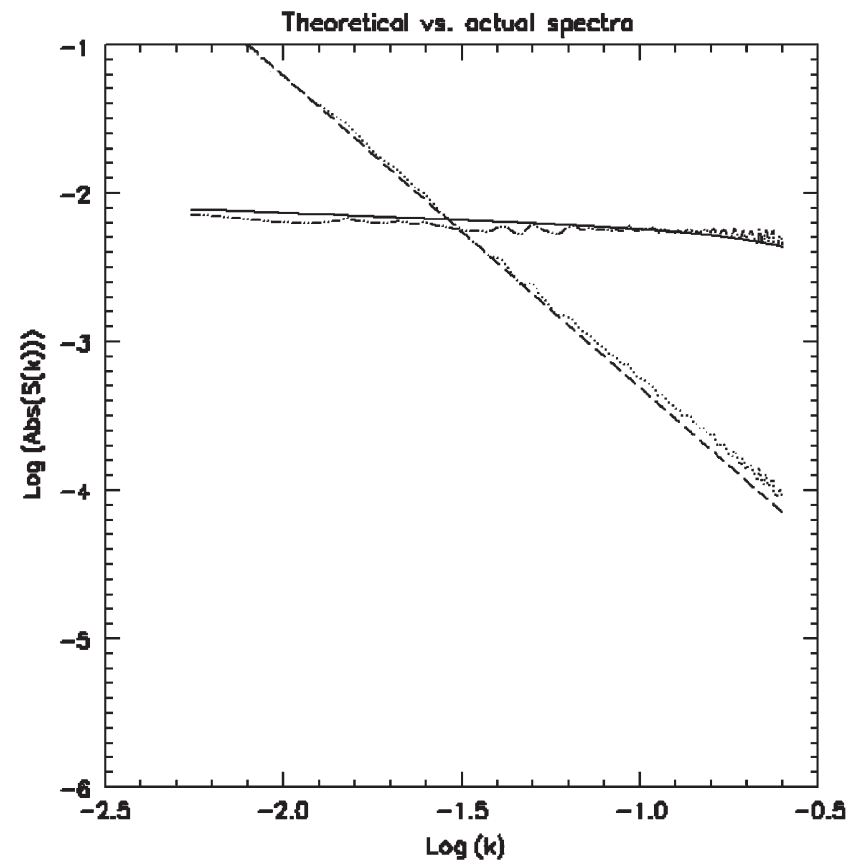

Fig. 8. Theoretical spectra of the surface (long dashed) and of the image (full line) versus estimated ones (dotted and dash dot, respectively). See case 6 of Table I for the employed parameters.

partly numerical approach has been employed to present the general case.

In particular, the logical sequence, and its main outcome, followed in the first part of this paper is given as follows.

1) To solve the problem of the nondifferentiability of the $\mathrm{fBm}$, we introduced a smoothed version of the imaged profile; this version, with the kernel considered in the filtering step, leads to a finite-difference derivative.

2) We demonstrated that the resulting derivative process shows a power-law spectrum only asymptotically, for low spatial frequencies.

3) With the spectral exponent being out of the range of allowed values for fractal profiles, we obtained that a fractal dimension for the derivative process cannot be defined in a simple way, i.e., linking it to the value of the Hurst parameter of the profile.

4) We found the relation between the Hurst parameter of the profile $H$ and the spectral exponent relevant to its derivative process. This relation can be exploited for the retrieving of $H$.

To study the general-slope case, we developed and presented a numerical framework based on reliable direct geometric and electromagnetic fractal models, which allows the simulation of any fractal profile of prescribed fractal parameters along with the evaluation of the signal backscattered to any prescribed microwave radar. From this numerical study, it turns out that the spectral behavior of the backscattered signal is no longer a power-law one, a definition of the image fractal dimension is not straightforward, and in any case, it cannot be analytically related to the fractal dimension of the sensed profile. This numerical framework also allowed us to validate the theoretical results that we presented for the small-slope case.
The twofold approach that we presented leads to the conclusion that the self-affine parameter $H$ of radar images is constrained in a range of values that do not allow a simple fractal dimension evaluation. However, a relation between the fractal parameters of the original profile and the obtained image exists, and in the small-slope case, it was analytically obtained in closed form. Finally, in Section IV, we discussed how the obtained formula can be used in the definition and design of appropriate inversion techniques.

In conclusion, the models and the mathematical framework presented in this paper have provided a background to enhance the understanding of natural features behavior in radar data and to develop in the near future new model-based applications on remote sensing data relevant to natural scenes.

\section{REFERENCES}

[1] J. M. Lopez-Sanchez, J. D. Ballester-Berman, and Y. Marquez-Moreno, "Model limitations and parameters-estimation methods for agricultural applications of polarimetric SAR interferometry," IEEE Trans. Geosci. Remote Sens., vol. 45, no. 11, pp. 3481-3493, Nov. 2007.

[2] S. Aksoy, H. G. Akcay, and T. Wassenaar, "Automatic mapping of linear woody vegetation features in agricultural landscapes using very high resolution imagery," IEEE Trans. Geosci. Remote Sens., vol. 48, no. 1, pp. 511-522, Jan. 2010.

[3] C. Arai, N. Matsuda, and M. Shikada, "An application of remote sensing and REAL TIME GIS to digital map for local government," in Proc. IGARSS, 2003, pp. 4552-4554.

[4] G. Schumann, R. Hostache, C. Puech, L. Hoffmann, P. Matgen, F. Pappenberger, and L. Pfister, "High-resolution 3-D flood information from radar imagery for flood hazard management," IEEE Trans. Geosci. Remote Sens., vol. 45, no. 6, pp. 1715-1725, Jun. 2007.

[5] S. Voigt, T. Kemper, T. Riedlinger, R. Kiefl, K. Scholte, and H. Mehl, "Satellite image analysis for disaster and crisis-management support," IEEE Trans. Geosci. Remote Sens., vol. 45, no. 6, pp. 1520-1528, Jun. 2007.

[6] G. Franceschetti, A. Iodice, and D. Riccio, "A canonical problem in electromagnetic backscattering from buildings," IEEE Trans. Geosci. Remote Sens., vol. 40, no. 8, pp. 1787-1801, Aug. 2002.

[7] R. Guida, A. Iodice, D. Riccio, and U. Stilla, "Model-based interpretation of high-resolution SAR images of buildings," IEEE J. Sel. Topics Appl. Earth Observ. Remote Sens., vol. 1, no. 2, pp. 107-119, Jun. 2008.

[8] B. Mandelbrot, The Fractal Geometry of Nature. New York: Freeman, 1983.

[9] G. Franceschetti and D. Riccio, Scattering, Natural Surfaces and Fractals. Burlington, MA: Academic, 2007.

[10] K. Falconer, Fractal Geometry. Chichester, U.K.: Wiley, 1989.

[11] J. S. Feder, Fractals. New York: Plenum, 1988.

[12] G. Franceschetti, M. Migliaccio, and D. Riccio, "An electromagnetic fractal-based model for the study of the fading," Radio Sci., vol. 31, no. 6, pp. 1749-1759, Nov./Dec. 1996.

[13] C. Elachi, Introduction to the Physics and Techniques of Remote Sensing. New York: Wiley, 1987.

[14] A. Pentland, "Fractal-based description of natural scene," IEEE Trans. Pattern Anal. Mach. Intell., vol. PAMI-6, no. 6, pp. 661-674, Nov. 1984.

[15] P. Kube and A. Pentland, "On the imaging of fractal surfaces," IEEE Trans. Pattern Anal. Mach. Intell., vol. 10, no. 5, pp. 704-707, Sep. 1988.

[16] G. Franceschetti, A. Iodice, and D. Riccio, "Fractal models for scattering from natural surfaces," in Invited Chapter in Scattering, R. Pike and P. Sabatier, Eds. London, U.K.: Academic, Sep. 2001, pp. 467-485.

[17] G. Ruello, P. Blanco, A. Iodice, J. J. Mallorqui, D. Riccio, A. Broquetas, and G. Franceschetti, "Synthesis, construction and validation of a fractal surface," IEEE Trans. Geosci. Remote Sens., vol. 44, no. 6, pp. 14031412, Jun. 2006.

[18] B. B. Mandelbrot and J. W. Van Ness, "Fractional Brownian motions, fractional noises and applications," SIAM Rev., vol. 10, no. 4, pp. 422 437, Oct. 1968.

[19] G. Di Martino, A. Iodice, D. Riccio, and G. Ruello, "A novel approach for disaster monitoring: fractal models and tools," IEEE Trans. Geosci. Remote Sens., vol. 45, no. 6, pp. 1559-1570, Jun. 2007.

[20] G. Franceschetti, A. Iodice, M. Migliaccio, and D. Riccio, "Scattering from natural rough surfaces modeled by fractional Brownian motion 
two-dimensional processes," IEEE Trans. Antennas Propag., vol. 47, no. 9, pp. 1405-1415, Sep. 1999.

[21] M. V. Berry and Z. V. Lewis, "On the Weierstrass-Mandelbrot fractal function," Proc. R. Soc. Lond. A, Math. Phys. Sci., vol. 370, no. 1743, pp. 459-484, Apr. 1980.

[22] M. V. Berry and T. M. Blackwell, "Diffractal echoes," J. Phys. A, Math. Gen., vol. 14, no. 11, pp. 3101-3110, Nov. 1981.

[23] P. Flandrin, "On the spectrum of fractional Brownian motions," IEEE Trans. Inf. Theory, vol. 35, no. 1, pp. 197-199, Jan. 1989.

[24] G. Franceschetti, A. Iodice, and D. Riccio, "Scattering from dielectric random fractal surfaces via method of moments," IEEE Trans. Geosci. Remote Sens., vol. 38, no. 4, pp. 1644-1655, Jul. 2000

[25] I. M. Gelfand and G. E. Shilov, Generalized Functions. Burlington, MA: Academic, 1964.

[26] I. S. Gradshteyn and I. M. Ryzhik, Table of Integrals, Series and Products. New York: Academic, 1980.

[27] R. F. Voss, "Random fractal forgeries," in Fundamental Algorithms for Computer Graphics, R. A. Earnshaw, Ed. New York: Springer-Verlag, 1985, pp. 805-835.

[28] G. Korvin, "Is the optical image of a non-Lambertian fractal surface fractal?" IEEE Geosci. Remote Sens. Lett., vol. 2, no. 4, pp. 380-383, Oct. 2005.

[29] P. Addesso, S. Marano, R. Restaino, and M. Tesauro, "Correlation properties of signals backscattered from fractal profiles," IEEE Trans. Geosci. Remote Sens., vol. 45, no. 9, pp. 2859-2868, Sep. 2007.

[30] L. Tsang, J. A. Kong, and R. T. Shin, Theory of Microwave Remote Sensing. New York: Wiley, 1985.

[31] S. M. Kay, Modern Spectral Estimation. Englewood Cliffs, NJ: PrenticeHall, 1988.

[32] T. Austin, A. W. England, and G. H. Wakefield, "Special problems in the estimation of power-law spectra as applied to topographical modeling," IEEE Trans. Geosci. Remote Sens., vol. 32, no. 4, pp. 928-939, Jul. 1994.

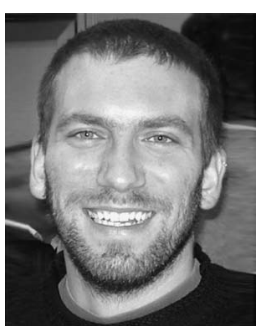

Gerardo Di Martino (S'06-M'09) was born in Naples, Italy, on June 22, 1979. He received the Laurea degree (cum laude) in telecommunication engineering and the Ph.D. degree in electronic and telecommunication engineering from the University of Naples "Federico II," Naples, in 2005 and 2009, respectively.

In 2009, he received a grant from the University of Naples to be spent at the Department of Biomedical, Electronic and Telecommunication Engineering for research in the field of indoor electromagnetic propagation. He is currently with the Department of Biomedical, Electronic and Telecommunication Engineering, working on a project aimed to the localization of unknown transmitters. His main research interests are in the field of microwave remote sensing and electromagnetics, which includes modeling of the electromagnetic scattering from natural surfaces and urban areas, SAR data simulation and information retrieval, and remote sensing techniques for developing countries.

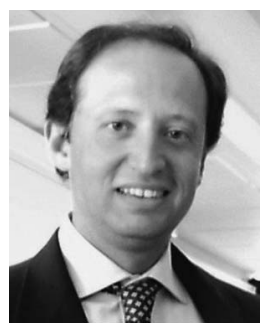

Antonio Iodice (S'97-M'00-SM'04) was born in Naples, Italy, on July 4, 1968. He received the Laurea degree (cum laude) in electronic engineering and the $\mathrm{Ph} . \mathrm{D}$. degree in electronic engineering and computer science from the University of Naples "Federico II," Naples, Italy, in 1993 and 1999, respectively.

In 1995, he received a grant from the Italian $\mathrm{Na}-$ tional Council of Research (CNR) to be spent at the Istituto di Ricerca per l'Elettromagnetismo e i Componenti Elettronici (IRECE), Naples, for research in the field of remote sensing. He was with Telespazio S.p.A., Rome, Italy, from 1999 to 2000. Since 2000, he has been with the Department of Biomedical, Electronic and Telecommunication Engineering, University of Naples "Federico II," where he is currently a Professor of electromagnetics. He is the author or a coauthor of about 170 papers published on refereed journals or on proceedings of international and national conferences. His main research interests are in the field of microwave remote sensing and electromagnetics, which include modeling of electromagnetic scattering from natural surfaces and urban areas, simulation and processing of synthetic aperture radar (SAR) signals, SAR interferometry, and electromagnetic propagation in urban areas.

Prof. Iodice was the recipient of the "2009 Sergei A. Schelkunoff Prize Paper Award" from the IEEE Antennas and Propagation Society.

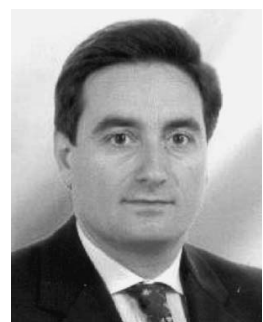

Daniele Riccio (M'91-SM'99) was born in Napoli, Italy, on April 13, 1962. He received the Laurea degree (cum laude) in electronic engineering at the University of Naples "Federico II," Naples, Italy, in 1989 .

$\mathrm{He}$ is a Professor of electromagnetics and remote sensing at the Department of Biomedical, Electronic and Telecommunication Engineering, University of Naples "Federico II." He was a Research Scientist at the Institute of Research on Electromagnetics and Electronic Components (IRECE) of the Italian National Council of Research (CNR). He is a member of the Cassini Radar Science Team. He also was a Guest Scientist at the German Aerospace Center (DLR) High-Frequency Institute, Munich, Germany, in 1994 and 1995, and a Visiting Professor at Universitat Politècnica de Catalunya, Barcelona, Spain, in 2006. $\mathrm{He}$ is the author of three books (including Scattering, Natural Surfaces and Fractals) and more than 220 published papers. He is an Associate Editor for Remote Sensing, Sensors, and The Open Remote Sensing Journal. His research interests are mainly focused on microwave remote sensing, synthetic aperture radar with emphasis on data simulation modeling and information retrieval for land oceanic and urban scenes, as well as on the application of fractal geometry to remote sensing and electromagnetic scattering from natural surfaces.

Prof. Riccio has received several fellowships from private and public companies (SIP, Selenia, CNR, CORISTA, CRATI) for research in the remote sensing field. He was the recipient of the 2009 Sergei A. Schelkunoff Prize Paper Award for the best paper published in year 2008 on the IEEE TRANSACTIONS ON ANTENNAS AND PROPAGATION.

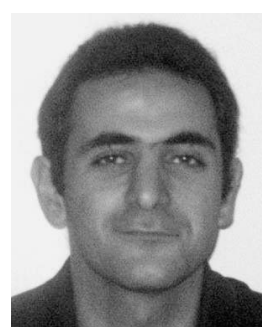

Giuseppe Ruello (S'00-M'04) was born in Naples, Italy, on February 12, 1975. He received the Laurea degree (with honors) in telecommunication engineering and the Ph.D. degree in information engineering from the University of Naples "Federico II," Naples, Italy, in 1999 and 2003, respectively.

In 2000, he received a grant from the University of Naples to be spent at the Department of Biomedical, Electronic and Telecommunication Engineering for research in the field of remote sensing. In 2000, he also received a grant from the University of Rome "La Sapienza," Rome, Italy. In 2002 and during 2004-2005, he was a Visiting Scientist at the Department of Signal Theory and Communications, Universitat Politecnica de Catalunya, Barcelona, Spain. He is currently a Research Scientist with the Department of Biomedical, Electronic and Telecommunication Engineering, University of Naples, Naples, Italy. He is the author of about 50 papers published on refereed journals or on proceedings of international and national conferences. His main research interests include SAR remote sensing, modeling of electromagnetic scattering from natural surfaces, SAR raw signal simulation, modeling of electromagnetic field propagation in urban environment, and remote sensing techniques for developing countries. 